\title{
AVALIAÇÃO COMPARATIVA DE RESULTADOS DE TESTES SENSORIAIS COM ESCALAS E TESTE DE ORDENAÇÃO PREFERÊNCIA COM CRIANÇAS E ADULTOS
}

\section{COMPARATIVE EVALUATION OF RESULTS OF SENSORY TESTS WITH SCALES AND ORDERNATION PREFERENCE TEST WITH CHILDREN AND ADULTS}

\author{
Ana Cristina da Silva Morais ${ }^{1}$; Lane de Lemos Cid ${ }^{1}$; Isadora Nolasco de Oliveira ${ }^{1}$; Maria do Carmo \\ Passos Rodrigues ${ }^{1}$ \\ ${ }^{1}$ Universidade Federal do Ceará - UFC - Fortaleza - Brasil crisanam@gmail.com
}

\begin{abstract}
Resumo
No presente estudo foram aplicados testes sensoriais afetivos com escalas e teste de ordenação preferência com crianças e adultos. Devido aos problemas envolvidos na aplicação da análise sensorial e na confiabilidade dos resultados obtidos com crianças estes foram comparados com os dos adultos. O objetivo foi avaliar a concordância de resultados do teste de aceitação global e do teste de ordenação preferência, tendo em vista que a partir da aceitabilidade podemos inferir a preferência, bem como verificar a capacidade das crianças de discriminar níveis de escalas sensoriais. Foram utilizadas três amostras de leite condensado de marca comercial designadas pelas siglas LC1, LC2 e LC3, correspondentes aos sabores tradicional, morango e chocolate. Os testes com escala aplicados foram escala hedônica e escala relativa ao ideal. Não houve concordância de resultados do teste de aceitação global e de ordenação preferência aplicados com crianças, enquanto nestes aplicados com adultos houve concordância. As crianças tiveram dificuldade de discriminar os níveis de gostar da escala hedônica apresentando pouca distribuição de notastanto para aceitação global como para aceitação do sabor. Os adultos discriminaram bem $e$ houve boa distribuição de notas entre os níveis da escala. No teste da escala relativa ao ideal, tanto crianças como adultos apresentaram boa distribuição de notas entre os níveis da escala. Com isso, percebe-se que estudos devem ser realizados com o intuito de definir as melhores condições $e$ métodos para que testes sensoriais realizados com crianças tenham resultados confiáveis.
\end{abstract}

Palavras-chave: testes sensoriais; crianças; adultos.

\section{Introdução}

Testes sensoriais com crianças têm se tornado cada vez mais importantes para a indústria de processamento de alimentos devido ao aumento do número de produtos direcionados ao mercado infantil e ao poder das crianças em decisões de compra. Contudo, as pesquisas sensoriais não têm sido encaminhadas para essa necessidade. Testes sensoriais com crianças são aplicados de modo 
limitado, entretanto ao longo dos anos, tem-se tornado evidente a importância de se conhecer o comportamento dessa população especial (KROLL, 1990).

O teste de ordenação é usado quando o objetivo é comparar várias amostras em relação a um simples atributo (como doçura, frescor) ou para avaliar a preferência; neste caso, duas ou mais amostras codificadas são apresentadas simultaneamente. Os julgadores são solicitados a ordená-las de acordo com sua preferência (KONKEL et al., 2004).

O teste de preferência pode ser considerado como uma das mais importantes etapas da Análise Sensorial. Representa o somatório de todas as percepções sensoriais e expressa o julgamento, por parte do consumidor, sobre a qualidade do produto. Os testes que podem ser empregados para determinação da preferência são teste pareado, teste de ordenação e escala hedônica (DUTCOSKY, 1996).

Os testes afetivos são utilizados quando se necessita conhecer o "status afetivo" dos consumidores com relação ao(s) produto(s), e para isso são utilizadas escalas hedônicas. Dos valores relativos de aceitabilidade pode-se inferir a preferência, ou seja, as amostras mais aceitas são as mais preferidas e vice-versa (KONKEL et al., 2004).

Kroll (1990) estudou a utilização de diferentes escalas de níveis em testes sensoriais com crianças. Foram utilizadas escala hedônica tradicional com nove pontos, escala facial e escala P\&K, desenvolvida pelo autor. A escala P\&K obteve melhor desempenho em relação à escala hedônica e à escala facial. Foi estudada também a interferência do comprimento da escala nos resultados. Observou-se que a escala de nove pontos foi tão boa, ou melhor, que a escala de sete pontos. O autor atribuiu ao fato de que escalas longas podem ser mais discriminadas e produzir resultados mais seguros.

Devido à importância do estudo da confiabilidade de dados sensoriais obtidos através de testes sensoriais com crianças, realizou-se o presente trabalho para avaliar a relação de resultados de teste de aceitação utilizando escala hedônica, de teste da escala relativa ao ideal e de ordenação preferência aplicados com crianças e fazer uma comparação da concordância desses resultados com os dos testes aplicados com adultos.

\section{Material e Métodos}


Os testes com crianças foram realizados numa Escola de Ensino Infantil, com alunos entre 4 e 12 anos de idade, através de entrevista individual (One-on-one interwiews). As condições para degustação foram aproximadas das condições laboratoriais. Os mesmos testes foram aplicados no laboratório de Análise Sensorial do Departamento de Tecnologia de Alimentos/UFC com adultos entre 19 e 31 anos. Em ambos os casos foram utilizados 42 provadores.

Foram utilizadas três amostras de leite condensado de marca comercial, designadas pelas siglas LC1, LC2 e LC3, correspondentes aos sabores tradicional, morango e chocolate, respectivamente. O delineamento utilizado foi de blocos completos balanceados e a apresentação das amostras foi de forma monádica seqüencial, para o teste de aceitação global e simultânea para o teste de ordenação preferência. As amostras foram servidas em copos plásticos, com quantidades padronizadas, e codificadas com três dígitos, obtidos de uma tabela de números aleatórios. Foi fornecida água a temperatura ambiente para limpeza do palato entre a avaliação das amostras (VICKERS, 1988; STONE, SIDEL, 1993). Utilizou-se a escala hedônica tradicional (1=desgostei muitíssimo; 5=nem gostei nem desgostei; 9=gostei muitíssimo) para avaliação da aceitação pelos adultos, segundo MEILGAARD et al. (1987) e a escala hedônica P\&K (1=detestei; 5=nem bom, nem ruim; 9=adorei) para a avaliação pelas crianças (KROLL, 1990). No teste de ordenação preferência pediu-se que cada provador ordenasse as amostras em ordem decrescente de preferência (MEILGAARD et al., 1999).

Os resultados da aceitação global foram avaliados através de histogramas de freqüência, análise de variância (ANOVA) e teste de médias Tukey $(\mathrm{p} \leq 0,05)$. Com os resultados do teste da escala relativa ao ideal foram feitos histogramas com o percentual de respostas por nível da escala. O teste de ordenação preferência foi avaliado através do teste de Friedman, utilizando a tabela de Newell e MacFarlane (DUTCOSKY, 1996).

\section{Resultados e Discussão}




\subsection{Teste de escala hedônica}

\subsubsection{Análise gráfica}

A Figura 1 representa os resultados obtidos na avaliação da aceitação global das três amostras, utilizando-se crianças como julgadores.

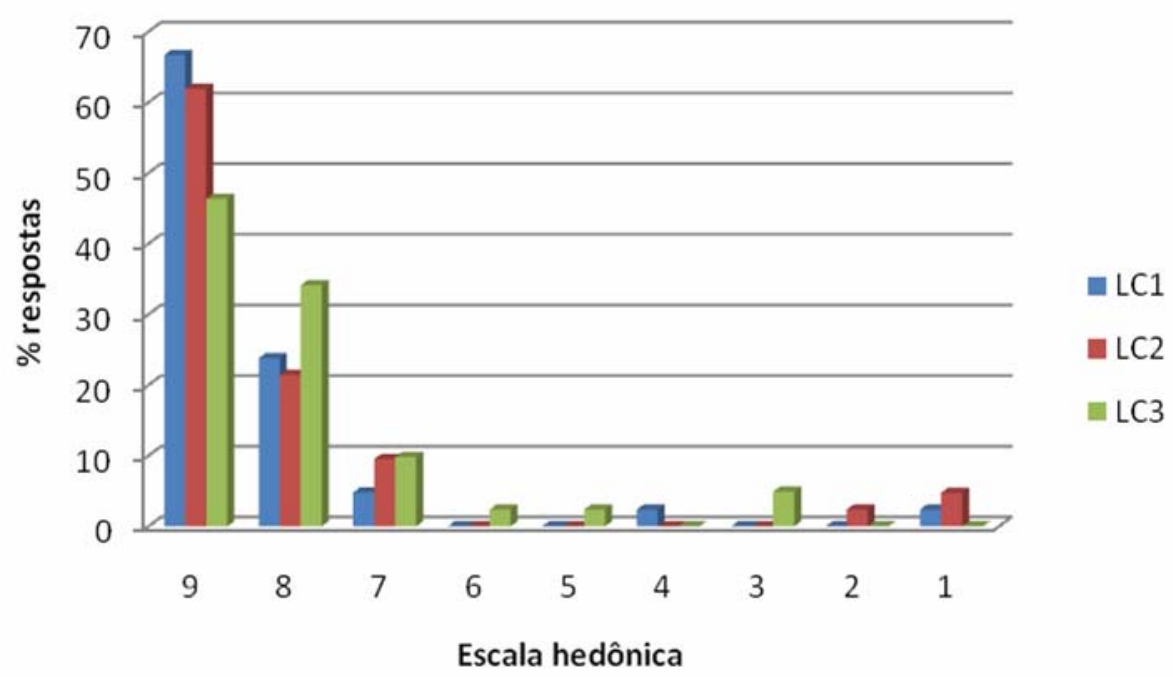

FIGURA 1. Freqüência das notas da escala hedônica para a aceitação global das amostras avaliadas por crianças.

$\mathrm{Na}$ avaliação da aceitação global pelas crianças, as três amostras alcançaram maior freqüência hedônica no nível 9 da escala, correspondente a "adorei”. Neste nível da escala a amostra LC1 alcançou $66,7 \%$ de respostas, a amostra LC2 61,9\% e a amostra LC3 46,3\%. O percentual de respostas na faixa de aceitação da escala foi de 95,3 para a amostra LC1; 92,8 para a amostra LC2 e 92,6 para a amostra LC3.

A Figura 2 representa os resultados obtidos na avaliação da aceitação global das três amostras, utilizando-se adultos como julgadores. 


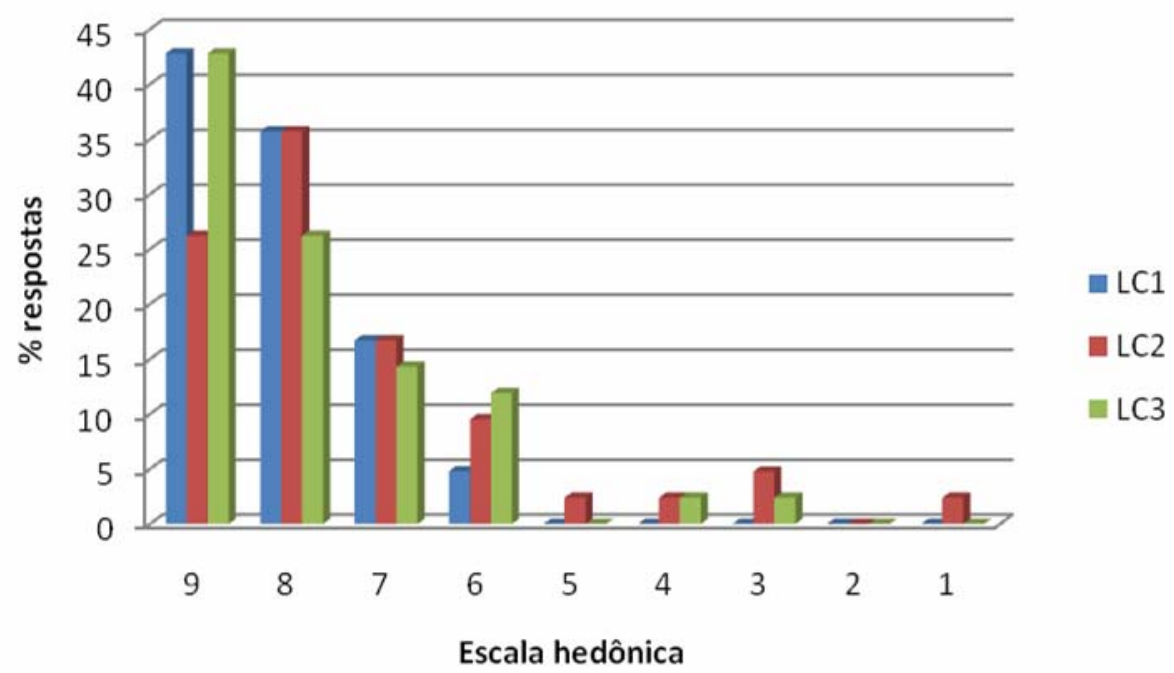

FIGURA 2. Freqüência das notas da escala hedônica para a aceitação global das amostras avaliadas por adultos.

De acordo com os resultados mostrados na Figura 2, as amostras LC1 e LC3 atingiram maior freqüência hedônica no nível 9 da escala ("gostei muitíssimo") com 42,8\% de respostas cada uma. A amostra LC2 alcançou maior percentual de respostas no nível 8 da escala ("gostei muito") atingindo um percentual de 35,7. Pela análise gráfica a amostra LC1 obteve $100 \%$ de respostas na faixa de aceitação da escala (9-6). A amostra LC3 obteve 95,2\% de respostas entre "gostei muitíssimo" e "gostei ligeiramente" e a amostra LC2 obteve 88,1\%.

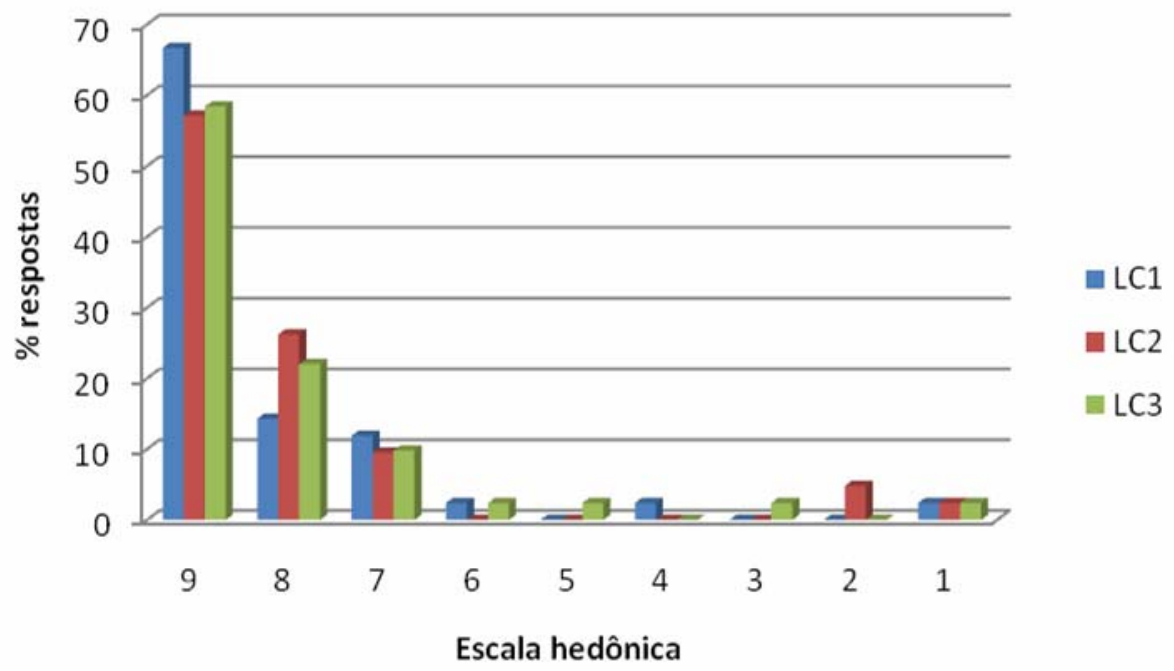

FIGURA 3. Freqüência das notas da escala hedônica para o atributo sabor das amostras avaliadas por crianças.

$\mathrm{Na}$ avaliação da aceitação do sabor pelas crianças, as três amostras obtiveram maior freqüência hedônica no nível 9 ("gostei muitíssimo"), sendo o percentual de respostas para as amostras LC1, LC2 e LC3, respectivamente; 66,7\%; 57, $1 \%$ e 58,4\%. 


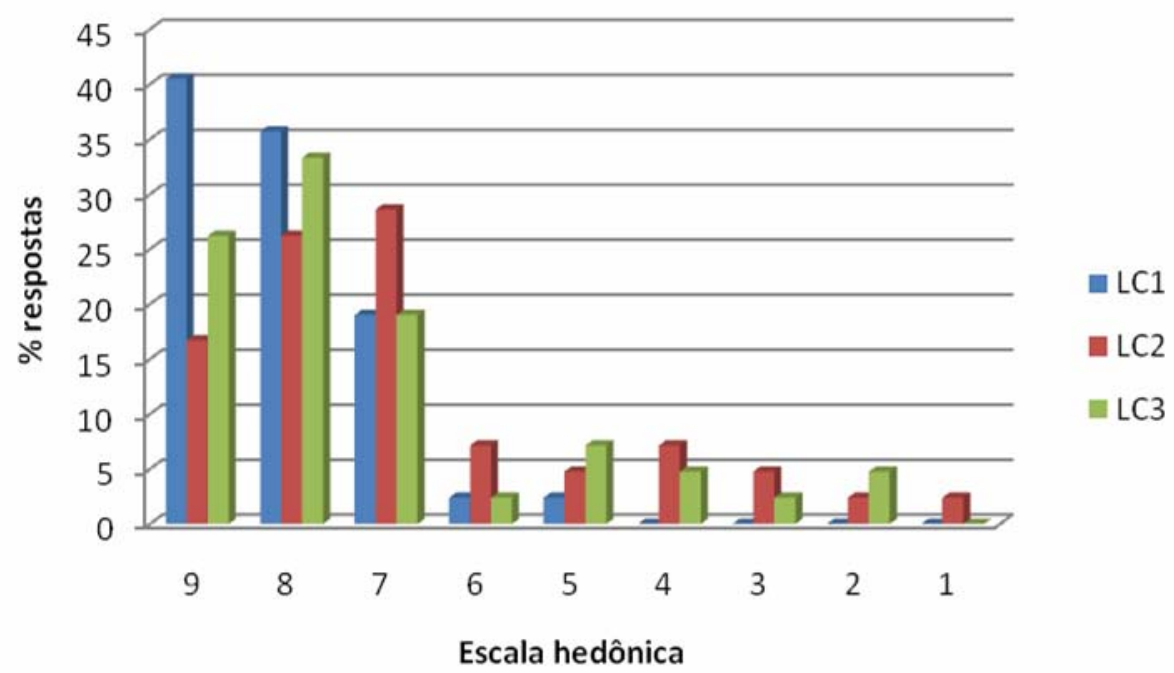

FIGURA 4. Freqüência das notas da escala hedônica para o atributo sabor das amostras avaliadas por adultos.

Nos resultados da avaliação do sabor pelos adultos, a amostra LC1 obteve maior freqüência hedônica no nível 9 (40,5\%), correspondente a "gostei muitíssimo". A amostra LC2 no nível 7 $(28,6 \%)$, correspondente a "gostei moderadamente" e a amostra 3 no nível 8 (33,3\%), correspondente a "gostei muito".

\subsubsection{Análise estatística}

Os resultados podem ser também avaliados pela Tabela 1, que apresenta as médias de aceitação das amostras em relação à aceitação global e ao atributo sabor avaliados por parte de crianças e adultos.

TABELA 1. Médias e desvios padrão das amostras para cada tipo de provador com relação à aceitação global e ao sabor.

\begin{tabular}{lcccc}
\hline & \multicolumn{2}{c}{ ACEITAÇÃO GLOBAL } & \multicolumn{2}{c}{ SABOR } \\
\cline { 2 - 5 } AMOSTRAS & Crianças & Adultos & Crianças & Adultos \\
\hline LC1 & $8,36 \pm 1,48^{\mathrm{a}}$ & $8,17 \pm 0,88^{\mathrm{a}}$ & $8,24 \pm 1,56^{\mathrm{a}}$ & $8,10 \pm 0,95^{\mathrm{a}}$ \\
LC2 & $8,05 \pm 2,00^{\mathrm{a}}$ & $7,33 \pm 1,84^{\mathrm{b}}$ & $8,02 \pm 1,91^{\mathrm{a}}$ & $6,76 \pm 2,02^{\mathrm{b}}$ \\
LC3 & $8,00 \pm 1,47^{\mathrm{a}}$ & $7,83 \pm 1,43^{\mathrm{ab}}$ & $8,07 \pm 1,69^{\mathrm{a}}$ & $7,21 \pm 1,93^{\mathrm{b}}$ \\
\hline
\end{tabular}

${ }^{\mathrm{a}, \mathrm{b}}$ Médias com letras iguais, em mesma coluna, não diferem entre si estatisticamente ao nível de 5\% de significância.

$\mathrm{Na}$ avaliação da aceitação global pelas crianças, não houve diferença significativa $(\mathrm{p} \leq 0,05)$ entre as médias das três amostras. No entanto, a maior média foi obtida pela amostra LC1 $(8,36)$. No resultado da avaliação pelos adultos, as amostras LC1 e LC2 diferiram entre si $(p \leq 0,05)$. A maior média obtida foi da amostra LC1 que alcançou 8,17. 
Os valores médios de aceitação na avaliação das crianças ficaram muito próximos, não apresentando diferença significativa $(\mathrm{p} \leq 0,05)$, sendo a amostra LC1 a mais aceita e a LC2 a menos aceita. Na avaliação dos adultos, apenas a amostra LC1 apresentou diferença significativa $(\mathrm{p} \leq 0,05)$ em relação às demais amostras, as quais não diferiram entre si.

\subsection{Teste da escala relativa ao ideal}

As Figuras 5 e 6 representam os resultados obtidos na avaliação da intensidade ideal da doçura das três amostras de leite condensado através da avaliação das crianças e dos adultos, respectivamente.

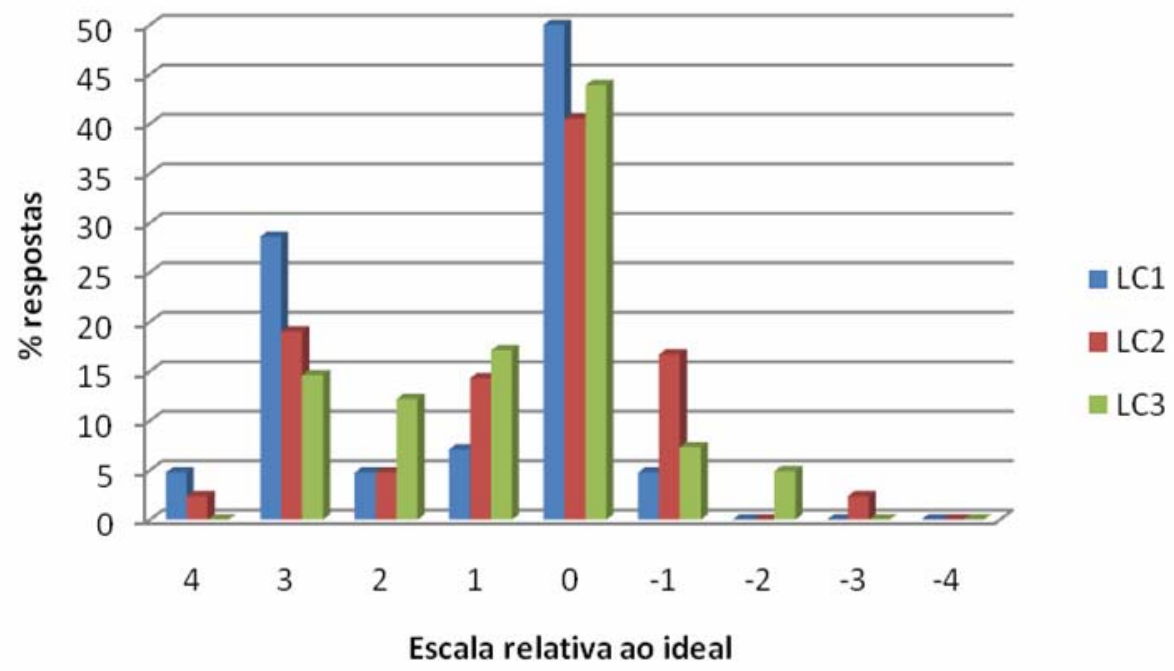

FIGURA 5. Freqüência das notas da escala relativa ao ideal para o atributo doçura pela avaliação das crianças.

A doçura da amostra LC1 atingiu 50\% de respostas equivalentes a ideal na avaliação das crianças, no entanto sua doçura encontra-se muito mais forte que o ideal por ter atingido $28,6 \%$ de respostas no nível " +3 " da escala. A amostra LC2 atingiu 40,5\% de respostas no nível zero da escala ("ideal"), porém sua doçura foi julgada muito mais forte que o ideal por ter alcançado 19\% de respostas no nível “+3”. Mesmo tendo atingido 43,9\% de respostas equivalentes a ideal, a doçura da amostra LC3 encontra-se ligeiramente mais forte que o ideal devido ter alcançado $17,1 \%$ de respostas no nível "+1” da escala. 


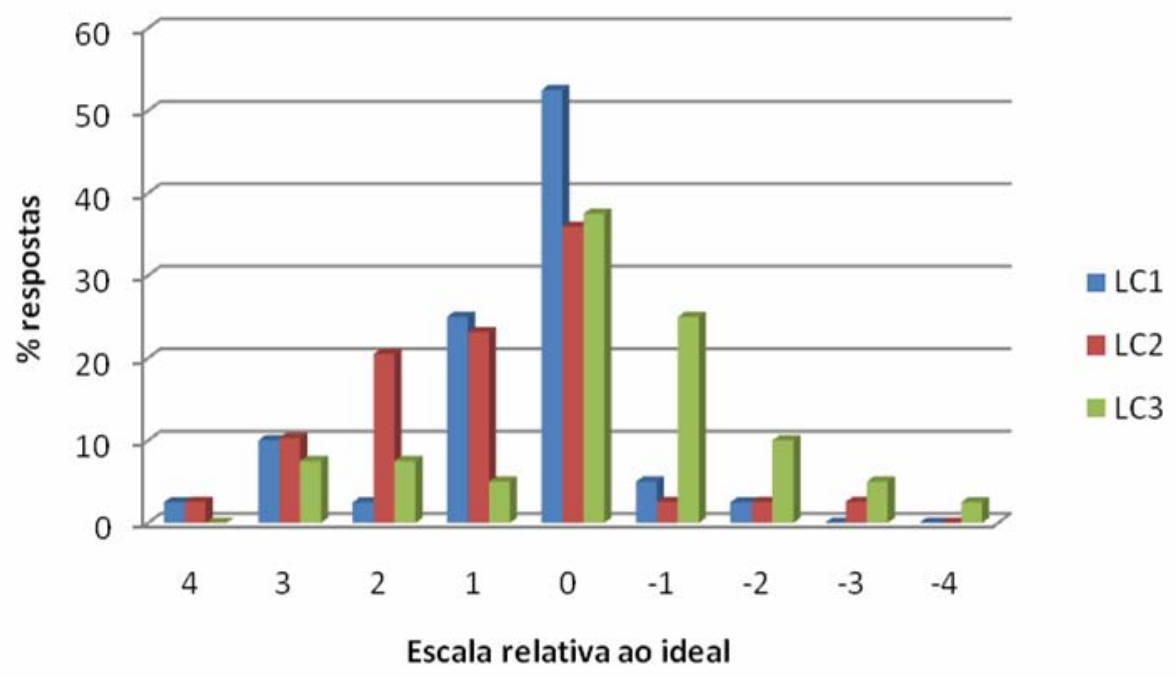

FIGURA 6. Freqüência das notas da escala relativa ao ideal para o atributo doçura pela avaliação dos adultos.

A doçura da amostra LC1 foi avaliada por 52,5\% dos adultos como ideal, no entanto encontra-se ligeiramente mais doce que o ideal (25\%). A amostra LC2 alcançou 35,9\% de respostas correspondentes ao ideal, mas encontra-se ligeiramente mais doce que o ideal (23,1\%). A doçura da amostra LC3 foi considerada ideal por 37,5\% dos provadores, no entanto encontra-se ligeiramente menos doce que o ideal (25\%).

\section{Teste de ordenação preferência}

Os resultados da avaliação sensorial no teste de ordenação feitos com dois tipos de provadores são mostrados na Tabela 2, Figura 7 e Figura 8.

TABELA 2. Avaliação de preferência por ordenação utilizando-se adultos e crianças.

\begin{tabular}{lcc}
\hline DIFERENÇA DA & \multicolumn{2}{c}{ MÓDULOS DA DIFERENÇA } \\
\cline { 2 - 3 } SOMA DE ORDENS & Orianças & Adultos \\
\hline LC1 - LC2 & $21(\mathrm{~ns})$ & 33 \\
LC1 - LC3 & $7(\mathrm{~ns})$ & $9(\mathrm{~ns})$ \\
LC2 - LC3 & $14(\mathrm{~ns})$ & 24 \\
\hline
\end{tabular}

(ns) = não significativo $(\mathrm{p} \leq 0,05)$.

No teste de ordenação feito por crianças, comparando-se os módulos da diferença com a DMS (diferença mínima significativa) percebe-se que não houve diferença estatística significativa $(\mathrm{p} \leq 0,05)$ entre as amostras. 
$\mathrm{Na}$ avaliação pelos adultos, não houve diferença significativa $(\mathrm{p} \leq 0,05)$ apenas entre as amostras LC1 e LC3. Observando-se o valor da diferença, podemos notar que amostra LC1 foi a mais preferida e a amostra LC2 a menos preferida.

$\mathrm{Na}$ avaliação da preferência pelas crianças não houve diferença significativa $(p \leq 0,05)$ entre nenhuma das amostras, enquanto na avaliação dos adultos apenas as amostras LC1 e LC3 não diferiram entre si. Com isso, percebe-se que as crianças não tiveram boa capacidade de discriminação.

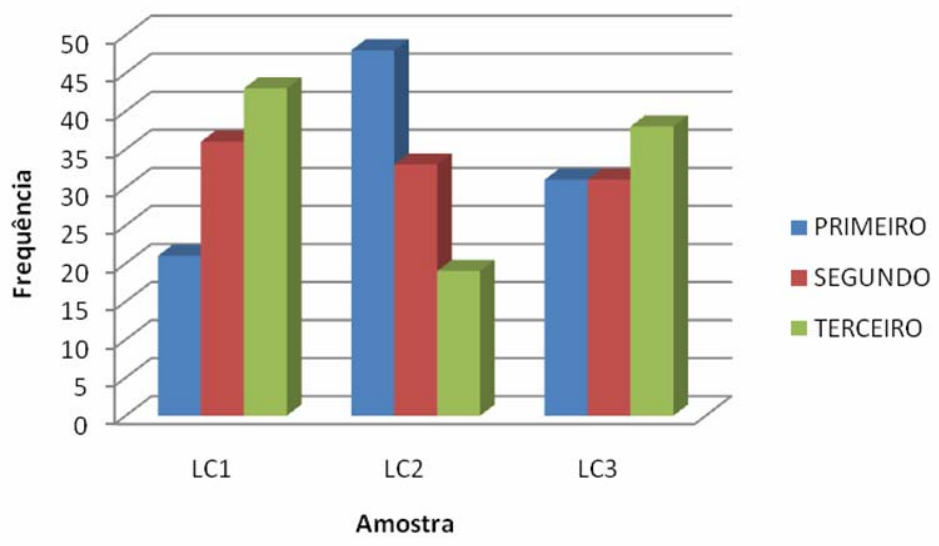

FIGURA 7. Freqüência das ordens das amostras pela avaliação das crianças.

De acordo com os resultados mostrados observa-se que a amostra LC2 ficou em primeiro lugar o maior número de vezes, sendo a amostra mais preferida pelas crianças. A amostra LC1 foi a menos preferida pelas crianças, pois ficou o menor número de vezes em primeiro lugar e o maior número de vezes em terceiro lugar.

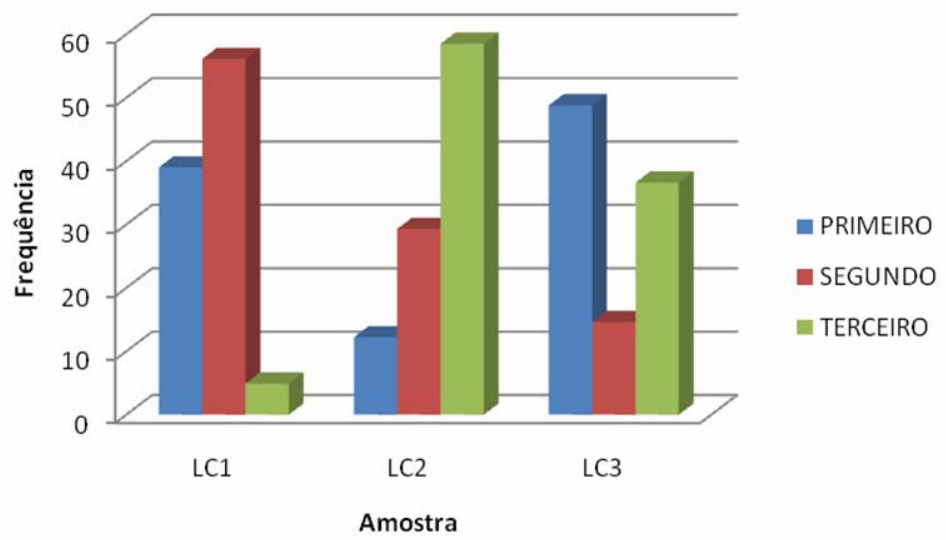

FIGURA 8. Freqüência das ordens das amostras pela avaliação dos adultos.

No teste de ordenação feito com adultos, a amostra LC3 foi a mais preferida ficando o maior número de vezes em primeiro lugar. A amostra LC1 ficou o maior número de vezes em segundo 
lugar enquanto a amostra LC2 ficou o maior número de vezes em terceiro lugar, sendo esta a amostra menos preferida pelos adultos.

Não houve concordância de resultados do teste de aceitação global e de ordenação preferência aplicados com crianças. Na avaliação da aceitação global a amostra de maior aceitação foi a LC1 e a menos aceita foi a LC3, enquanto no teste de ordenação preferência a amostra mais preferida foi a LC2 e a menos preferida a LC1. Os resultados dos dois testes deveriam ser concordantes, pois a partir da aceitabilidade podemos predizer a preferência, ou seja, as amostras mais aceitas são as mais preferidas e vice-versa (KONKEL et al., 2004).

$\mathrm{Na}$ avaliação dos adultos os resultados foram concordantes. A amostra LC1 foi tanto a mais preferida como a mais aceita e a amostra LC2 foi menos preferida e menos aceita.

\section{Conclusões}

As crianças mostraram dificuldade em discriminar os níveis de gostar da escala. Não houve concordância de resultados dos testes de escala hedônica (aceitação global) e de ordenação preferência feito pelas crianças, uma vez que no primeiro a amostra de maior aceitação foi a LC1 e a de menor a LC3 e no segundo a mais preferida foi a LC2 e a menos preferida a LC1. No teste com adultos houve concordância de resultados, sendo a amostra LC1 a mais preferida e a LC2 a menos preferida nos dois testes. A concordância de resultados é esperada tendo em vista que através da aceitação podemos predizer a preferência.

$\mathrm{Na}$ avaliação da aceitação do sabor as crianças também mostraram dificuldade em discriminar os níveis de gostar da escala, pois as três amostras alcançaram maior freqüência hedônica no nível 9 da escala ("adorei”) enquanto os adultos apresentaram boa distribuição das respostas entre os níveis de gostar da escala (9 "gostei muitíssimo"- 6 "gostei ligeiramente"). Houve concordância de resultados dos testes de aceitação de sabor (escala hedônica) avaliada por crianças e adultos, haja vista que em ambos os grupos de avaliadores a amostra LC1 foi a preferida (maior média de aceitação) e a amostra LC2 a menos preferida (menor média de aceitação) em sabor.

No teste da escala relativa ao ideal, os histogramas de freqüência dos resultados da avaliação feita pelas crianças apresentaram boa distribuição das respostas, tendo entre seis e sete níveis com respostas; enquanto os histogramas da avaliação dos adultos apresentaram entre quatro e seis níveis 
com respostas. Com isso, percebe-se que as crianças conseguem discriminar níveis da escala relativa ao ideal.

Os resultados obtidos mostram que pesquisas devem ser realizadas com o intuito de definir as melhores condições e métodos para que testes sensoriais realizados com crianças tenham resultados confiáveis.

\begin{abstract}
In this work affective sensory tests with scales and preference ordinance test had been applied with children and adults. Due to the problems involved in the application of the sensory analysis and in the trustworthiness of the results gotten with children these had been compared with the ones of the adults. The objective was to evaluate the agreement of results of the global acceptance test and of the ordinance preference test, in view that from acceptability we can infer the preference, as well as verifying the capacity of the children to discriminate levels of sensory scales. The tests with scale applied had been hedonic scale and relative scale to the ideal. It did not have agreement of results of the global acceptance test and ordinance preference test applied with children, while in these applied with adults it had agreement. The children had difficulty in discriminating the levels to like of the hedonic scale presenting little note distribution for global acceptance as for flavor acceptance. The adults discriminated well and had good note distribution between the levels of the scale. In the test of the relative scale to the ideal, as many children as adult had presented good note distribution between the levels of the scale. With this, one perceives that studies must be carried through in order to define the best conditions and methods so that carried through sensorial tests with children have reliable resulted.
\end{abstract}

Key-words: sensory tests; children; adults.

\title{
5. Referências
}

DUTCOSKY, S. D. Análise sensorial de alimentos, Curitiba: CHAMPAGNAT, 1996.

KONKEL, F. E.; OLIVEIRA, S. M. R.; SIMÕES, D. R. S.; DEMIATE, I. M. Avaliação sensorial de doce de leite pastoso com diferentes concentrações de amido. Ciênc. Tecnol. Aliment., v. 24, n. 2, p. 249-254, 2004.

KROLL, B. J. Evaluating rating scales for sensory testing with children. J. Food Technology., v. ,n. , p. 78-86, 1990.

MACHADO, E. C.; FERREIRA, C. L. L. F.; FONSECA, L. M.; SOARES, F. M.; PEREIRA JÚNIOR, F. N. Características físico-químicas e sensoriais do queijo minas artesanal produzido na região do serro, Minas Gerais. Ciênc. Tecnol. Aliment., v. 24, n. 4, p. 516-521, 2004.

MEILGAARD, M., CIVILlE, G.V., CARR, B.T. Sensory evaluation techniques. $3^{\text {rd }}$. ed., New York: CRC Press, 1999, 387p.

MEILGAARD, M.; CIVILLE, G. V.; CARR, B.T. Sensory evaluation techniques. Florida: CRC Press Inc., 1987. v. II. $158 \mathrm{p}$.

OliVEIRA, A. P. V.; FRASSON, K.; ALMEIDA, T. C. A.; BENASSI, M. T. Aceitação de sobremesas lácteas dietéticas e formuladas com açúcar: Teste afetivo e mapa de preferência interno. Ciênc. Tecnol. Aliment., v. 24, n. 4, p. 627-633, 2004. 
SANTANA, L. R. R.; MATSUURA, F. C. A. U.; CARDOSO, R. L. Genótipos melhorados de mamão (Carica papaya L.): Avaliação sensorial e físico-química dos frutos. Ciênc. Tecnol. Aliment., v. 24, n. 2, p. 217-222, 2004.

STONE, H.; SIDEL, J. L. Sensory evaluation practices. 2nd ed. London: Academic Press, 1993. 338 p.

TUNALEY, A., THOMSON, D.M.H., McEWAN, J.A. Determination of equi-sweet concentrations of nine sweeteners using a relative rating technique. International Journal of Food Science and Technology, v. 22, p. 627-351 1987.

VICKERS, Z. Sensory specific satiety in lemonade using a just right scale for sweetness. Journal of Sensory Study, V.3, n1, p.1-8, 1988.

\section{Dados do primeiro autor:}

Nome completo: Ana Cristina da Silva Morais*; Isadora Nolasco de Oliveira; Lane de Lemos Cid; Maria do Carmo Passos Rodrigues

Filiação institucional: Universidade Federal do Ceará - UFC

Departamento: Departamento de Tecnologia de Alimentos (Laboratório de Análise Sensorial)

Função ou cargo ocupado: Aluna de pós-graduação*, alunas de graduação e docente.

Endereço completo para correspondência: Avenida Mr. Hull, 2977 Bloco 857 Alagadiço Fortaleza, CE - Brasil Caixa-Postal: 12168 CEP: 60356-000

Telefones para contato: *(85) 3491-2405

e-mail: crisanam@gmail.com 